\title{
When situativity meets objectivity in peer-production of knowledge: the case of the WikiRate platform
}

Richard Mills

Stefano De Paoli

This is the Author Accepted Manuscript. The final published version is available at Emerald via doi: https://dx.doi.org/10.1108/DTA-02-2017-0006 
When situativity meets objectivity in peer-production of knowledge: the case of the WikiRate platform

Richard Mills - University of Cambridge (rm747@cam.ac.uk)

Stefano De Paoli - Abertay University (‥depaoli@abertay.ac.uk)

\section{Abstract}

- Purpose: The purpose of this paper is to further the debate on Knowledge Artifacts, by presenting the design of WikiRate, a Collective Awareness platform whose goal is to support a wider public contributing to the generation of knowledge on Ethical, Social and Governance performance of companies.

- Approach: The material presented in the paper comes from first-hand experience of the authors as part of the WikiRate design team. This material is reflexively discussed using concepts from the field of Science and Technology Studies.

- Findings: Using the concept of the 'funnel of interest' we discuss how the design of a Knowledge Artifact like WikiRate relies on the designers' capacity to translate general statements into particular design solutions. We also show how this funnelling helps understanding the interplay between situativity and objectivity in a Knowledge Artifact. We show how WikiRate is a peerproduction platform based on situativity, which requires a robust level of objectivity for producing reliable knowledge about the Ethical, Social and Governance performance of companies.

- Originality/value: This paper furthers the debate on KAs. It presents a relevant design example and offers in the discussion a set of design and community building recommendations to practitioners.

Keywords: peer-production, crowdsourcing, Corporate Social Responsibility, funnel of interest, situativity, objectivity 


\section{INTRODUCTION}

The goal of this paper is to present a first-hand account and a direct experience of designing an Information Technology Knowledge Artifact (ITKA) - the WikiRate platform. Starting from the Wikirate design experience our main contribution is to conceptualise elements of novelty for the debate about Knowledge Artifacts (KAs), artifacts purposely built to support knowledge-related processes. These elements of novelty are also crystallised, in the discussion, in a number of practical recommendations based on our direct design experience, which can support design and community building in application areas similar to that of the WikiRate project.

The WikiRate project's tagline is "crowdsource better companies" and this bakes in two fundamental constraints - the subject and means of production of the KA further solidified by the fact that the project is funded under the European Commission Framework Programme 7 area "Collective Awareness Platforms for Sustainability and Social Innovation" (CAPS). The platform's purpose is to make companies better, via "crowdsourcing" knowledge about their Environmental, Social and Governance (ESG) performance. WikiRate is also Free Libre Open Source Software (FLOSS) and offered to the public without restriction. The project proposal and the related Description of Work (DOW) document contain information about the project's approach, including specifying functional elements of the platform and roles of each partner institution. However, the design has often proceeded in a nonlinear fashion, occasionally re-interpreting the DOW in pursuit of "crowdsourcing better companies". This paper tells part of the story of this process. An interesting aspect is that the purpose of WikiRate is specified not in terms of the ESG knowledge itself, but in terms of how this knowledge should be used. The first question for the design team was therefore "what kind of knowledge can be used to improve the ESG performance of companies?" The answer to this question formed the basis of WikiRate's design (Mills et al., 2016).

A conceptual starting point to discuss WikiRate is the articulation between objectivity and situativity in KAs postulated by Cabitza and Locoro (2014) - which allows one to map (IT)KAs depending on the more or less situated or objective degrees to which knowledge is represented, stored and managed within an Information Technology for the subsequent use of i.e. an organisation. We believe that WikiRate presents novel elements for conceptualising the relation between design and user knowledge creation in ITKAs. In WikiRate knowledge creation is articulated starting from broad statements about the current state of ESG performance knowledge and how the platform should be positioned to make an intervention, down to particular design choices through which this positioning is concretised as software. To conceptualise this movement from general statements to particular design solutions, we reconsider a concept from Science and Technology Studies (STS): the 'funnel of interest' (Law, 
1986). This was proposed for studying how scientific papers funnel the interest of readers, starting with general hypotheses and then funnelling readers toward particular research elements (e.g. experiments) thus forcing readers to accept the paper's conclusions. With the 'funnel of interest' we show how the general WikiRate mission statement is translated into design solutions by which new knowledge about ESG performance is then created by users. This concept as applied to WikiRate also illuminates an interplay between situativity and objectivity. WikiRate is a wiki-like platform (based on the framework Wagn ${ }^{1}$ ), driven by user peer-production - and thus it could be considered moreso on the situativity side of Cabitza and Locoro's conceptualisation. However, Wikirate is based also on objective/representational goals. A key aim in designing WikiRate is to enable a situated peer production community to build robust and objective representations of ESG performance knowledge. The main contribution of the paper is a discussion of how we approached this problem and what has been learned from this experience, which could benefit other similar projects. What we have learned is presented in a set of recommendations for design and community building in the discussion section of the paper.

This paper is organised as follows: in section 2 we review relevant literature on organisational knowledge and Information Technology; in section 3, we introduce the funnel of interest as our interpretative framework; in section 4 and 5 we present our design experience of Wikirate as a Knowledge Artifact; section 6 presents a discussion of the findings, concluding by offering practical recommendations.

\section{KNOWLEDGE, ORGANISATIONS AND IT ARTIFACTS}

To better frame the KAs debate we start by considering some classical positions in the debate on organisational knowledge and the use of Information Technologies (ITs). We consider organisational knowledge as an accumulation of past experience based on routine practices which can in turn be mobilised for future behavior within organisations (Levitt \& March, 1988). It is thus the knowledge which an organisation has learned with experience and which uses for organisational action. This debate on organisational knowledge - at a simplified level - sees two polar opposites. On one side, we have the idea that routinised knowledge can be objectified, made manageable, measurable and controllable within organisations, especially via ITs. On the other side, we have the idea that knowledge is produced largely by doing things and it remains mostly subjective, fuzzy and context dependent. It is, in other words, much less formalisable within IT constructs. The perspective by Davenport et al. (1988) on Knowledge Management offers an influential example of the first position when they argue that organisations should: Create knowledge repository,

\footnotetext{
1 http://wagn.org/
} 
Improve knowledge assets, Enhance the knowledge environment and, Manage knowledge as an asset (p. 44-45). At the opposite side are the practice based studies in organisational learning, where practice is a notion that connects 'knowing' and 'doing' (Gherardi, 2000). The emphasis is not on knowledge as a manageable object but on the process of 'knowing something by doing' within communities of practice (Wenger, 2000). However, this polarisation between knowledge in practice and knowledge as a formal asset is not fully exhaustive as there may be overlaps (Norman, 1993). A widely known literature study of these overlaps comes from Nonaka and Takehuci (1995) which showed that there is often a dynamic interaction at several levels - Socialisation, Externalisation, Combination and Internalisation between tacit and explicit knowledge, which helps explaining how knowledge is created in practice and then used in organisations.

The debate on the use of ITs in organisational knowledge embraces at least two relevant aspects: one is the role of ITs in the formalisation and management of organisational knowledge and the second relates to the design of ITs used in organisations.

Historically, when it comes to consider these issues an interesting area is that of Artificial Intelligence (Al). Originally much of this field has been shaped around the idea that software could be conceptualised around the Cartesian principle of a thinking substance (Sharples et al. 1989) and that software (and intelligent/adaptive systems), like human beings should be considered manipulators of symbols. An Al is thus directed to the realization of an end or adaptation to an environment, achieved with problem solving and response to environmental stimuli (Simon, 1996). This can be seen also in the implementation and use of Information Systems (ISs) where especially management ones - are designed for achieving a specific purpose within a defined environment in a so-called design-science (Hevner et al, 2005). Management sets these purposes for the system, establishing measurable objectives, along with feedback methodologies to monitor the results. Traditionally the design methodologies of ITs assume a linear form, divided into sequential steps (Avison and Fitzgerald, 1995). The implementation and development of software, thus follows a scientific organization of labor, where the various elements of a system are chosen based on their contribution to achieve its (managerial) purpose. This also relates to controlling the elements of the systems - including end-users so that they can be aligned to the managerial objectives.

In the field of Al, Winograd and Flores (1986) criticized the excessive rationalism and push toward objectivity of the discipline and introduced a perspective for developing intelligent systems grounded in situational context and using practical knowledge. Suchman (1987) introduced the concept of situated action criticising the perspective of designing interactive systems based on decoupling intelligence from artifacts, leading to the production of formal models of knowledge. Suchman argued against 
designing technologies around plans where the action is a linear sequence of steps to accomplish a set objective. For Suchman, an action is situated (even when the IT design is based on plans) and thus depends on its material and social circumstances. The phenomenological perspective also argues for a shift from control to "Drift" (Ciborra and associates, 2000). Control is exercised by managers on their own organizations, by means of ITs implemented following managerial principles. An alternative conception is proposed where the successful introduction of a new technology should be based on improvisation and bricolage (Ciborra, 2002).

Before presenting our research we need to briefly recap some new developments as well as the evolution of some concepts. In the limited space of this manuscript we focus on the emergence of produsage/peer production.

Development methodologies have seen a partial shift from structured ones toward agile approaches, with a leaner structure capable of answering unforeseen challenges and variations based on early releases, adaptive learning and continuous improvements. This approach also places emphasis on people (Cockburn and Highsmith, 2001), on their skills and abilities as problem solvers. Agile approaches resonate well with a partial shift in the organisation of software development teams, initially in FLOSS projects, where relatively large and distributed teams approach development in an agile way rather than having a smaller and closed team organised with scientific management. In a classical paper, Raymond (1998) used the metaphor of the Cathedral as opposed to the Bazaar to present this shift. In FLOSS we have multiple cultures and a collective set of practices that are not fixed, but rather, indeterminate, flexible and contestable (Lin, 2005). FLOSS is based on distributed intelligence (Kogut and Metiu, 2001) and organisational efforts are coordinated with artifacts such as software licenses (Lanzara and Morner, 2005) or code repositories (De Paoli and D'Andrea, 2008). This in part also leads to produsage: a shift in production processes (for e.g. media content) where users create content in a variety of online environments, characterised by voluntary participation, fluid roles and collective ownership (Bruns, 2008). Products of produsage are open ended and under continuous development, whereas products in traditional production are closed and subject to long cycles of revisions. Produsage projects may also use strategies such as voting mechanisms to 'filter' the sheer amount of content being produced (Mills \& Fish, 2015).

In terms of peer-production, Haythornthwaite (2009) has identified two models which depend on the level of freedom that users can exercise. She distinguishes between lightweight (LWPP) and heavyweight peer-production (HWPP). In the first "Knowledge of what to do and how to contribute are defined by authorities or owners of the enterprise in such a way that contributors can easily begin to provide input". Whereas, the second case "involves not only contributions to the product, but also 
attention to the actions and contributions of others, and a commitment to maintaining and sustaining the direction and viability of the community". LWPP and HWPP are not necessarily mutually exclusive, and hybrid solutions may be observed. In a popular contribution, Howe (2006) defined crowdsourcing as "the act of taking a job traditionally performed by a designated agent (usually an employee) and outsourcing it to an undefined, generally large group of people in the form of an open call." Whereas commons-based peer production is associated with the creation of a shared resource under collective stewardship, crowdsourcing may involve being paid for the completion of work by an organisation that owns the product of that work.

\section{INTERPRETATIVE FRAMEWORK}

We present here our own experience in the design of the WikiRate platform as a KA, firstly via the work of Cabitza and Locoro (2014). The authors reviewed a substantial body of literature on KAs and offered an analytical view of different definitions and an interpretative framework for mapping KAs based on the categorical dimensions of situativity and objectivity. Situativity is "the extent the $K A$ is capable to adapt itself to the context and situation at hand, as well as the extent it can be appropriated by its users and exploited in a given situation."(p. 576), whereas objectivity is " the capability of the KA to represent true facts in an objective, crisp, and contextindependent manner, as well as the extent it can be transferred among its users as an object carrying some knowledge with itself." (p. 576).

Cabitza and Locoro place their categories in a perpendicular relation and map KAs, presenting situations in which KAs have differing degrees of situativity and objectivity (Figure 1). For instance Al applications, such as expert systems based on representational knowledge, possess a high degree of objectivity and little situativity. Knowledge Management (KM) tools similarly present a high level of objectivity in relation to the managerial needs of controlling organisational knowledge, however they also present some degree of situativity insofar as knowledge can be transferred to individuals supporting their routine actions. On the opposite side, Computer Supported Cooperative Work (CSCW) projects and (to a lesser degree) Computer Supported Cooperative Learning (CSCL), in which we may also recognise peerproduction efforts (e.g. Wikis), are flexible and can be appropriated almost entirely by users to various ends, but as a result may lack rigidly defined objectivity. Information Systems sits in between objectivity and situativity presenting a balanced situation. 


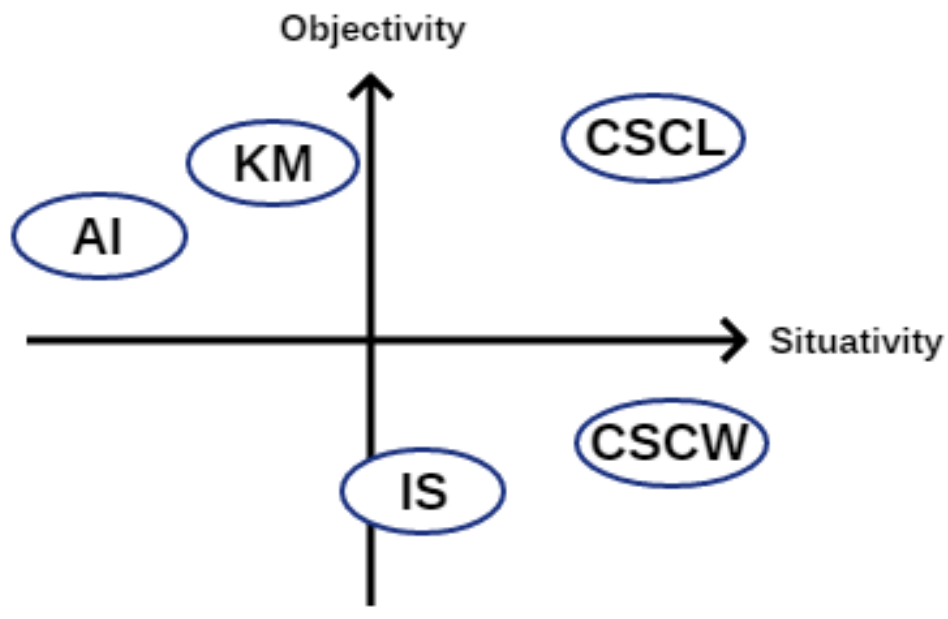

Figure 1 - The mapping of KAs adapted from Cabitza and Locoro (2014)

Cabitza and Locoro end their paper by hinting at the possibility that their categorical dimensions should be augmented by inquiring into specific design principles, design solutions or requirements for KAs. This is one of the contributions we will make by presenting the WikiRate design, considering that it is a peer-production platform which presents peculiar objective stances.

Our contribution considers how knowledge is being created with a KA and how this connects with the design of the KA. We call this dimension 'funnelling' and discuss how a KA design - at least in our experience of designing WikiRate - can be interpreted as a translation of general mission statements and requirements into specific design solutions. We approach the description of our own experience of the $\mathrm{KA}$ design also in a reflexive manner since we have been involved in design processes and decision making for WikiRate. Thus in this paper we also offer our first-hand views and reflections of (some) design decisions: our own reflexivity is part of the construction of technology in action (Latour, 1987).

In the STS tradition a relevant concept to understand this type of reflexivity is that of inscription, where "An inscription is the result of the translation of one's interest into material form (Callon 1991, 143). For Akrich $(1992,208)$ "A large part of the work of innovators is that of "inscribing" this vision of (or prediction about) the world in the technical content of the new object". Designers shape a new technological innovation by adding scripts - translations of one's personal interest or vision of how the world ought to be - with a focus on anticipating various (human and non-human) actors' uses - thus interesting them in the innovation. With inscriptions, designers also offer a prediction on how knowledge production will be stabilised via said innovation.

In line with the above, an interesting but overlooked concept is that of the 'funnel of interest' (Law, 1986). We re-interpret this concept in order to account for the process 
of knowledge creation in WikiRate. Technology innovators need to interest and enrol a number of actors/entities - humans and non-humans - to make their innovation viable. This is done by adding inscriptions which cultivate interest in entities so that they will become part of the innovation. Classical examples of this are that of the Hotel Manager by Latour (1991), using a metal weight to force customers to bring back room keys or that of scientists using a net to prevent predators eating scallops (Callon, 1984).

The 'funnel of interest' was proposed to understand how a scientific paper forces readers to accept scientific conclusions, which in turn support a scientific hypothesis. Law postulates that this is achieved by the author(s) first starting with very general statements (i.e. inscriptions) about what one wants to achieve and from this the author seeks to funnel the interest of readers - with more detailed inscriptions toward the particular, via a reader acceptance of the research methodology and the results of experiments. The principle of the funnel of interest is "starting with a force that is generally respected and thence of translating the reader from general to the particular" (p. 77). Thus at the beginning "the mouth of such as funnel is broad in order to suck in as wide an audience as possible. Thereafter it narrows [...]" and "interests are channelled". (p. 77). It is an exercise of persuasion where starting with general statements and then funnelling the reader ensures that the reader approves the conclusion. This concept offers an interpretative lens for approaching certain aspects of KAs design. We will also reflexively expose some of the adjustments and redefinition that the WikiRate team went through in order to funnel the production of knowledge.

Figure 2 shows how we reinterpret Law's funnel of interest in the context of KAs: designers may start by postulating a very general mission statement about a certain problem in the world needing a solution (what Callon, 1984 calls problematisation). Designers then articulate this via specific and targeted statements or requirements (other inscriptions) in which social and technological elements/actors of the KA are further defined. These are in turn translated into design solutions which support actors in generating knowledge addressing the general mission statement. 


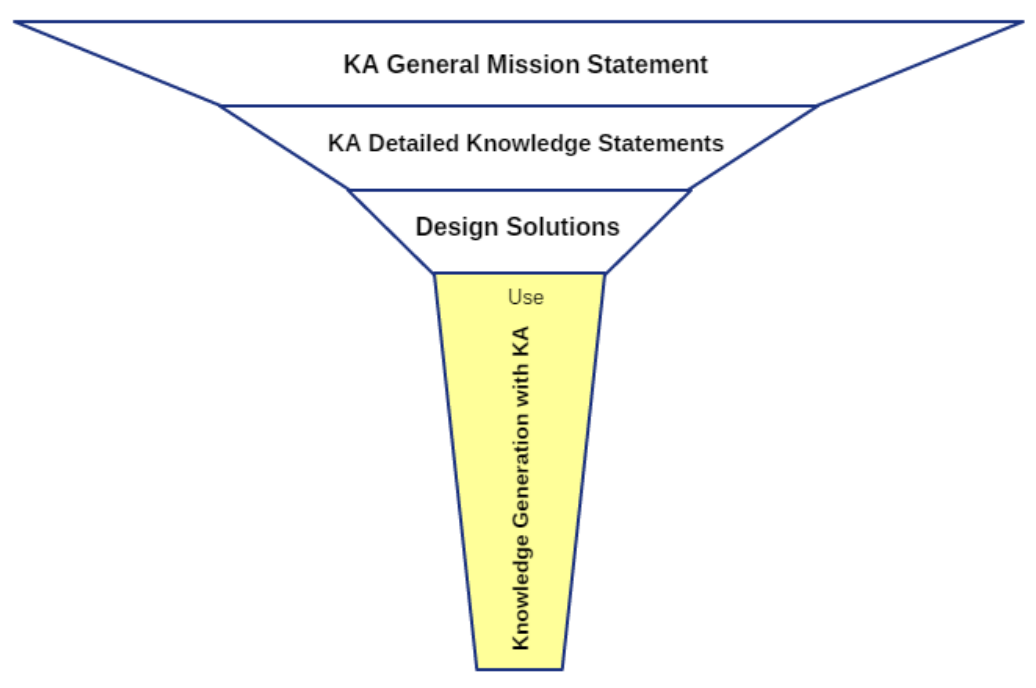

Figure 2 - The funnel of interest for a KA

\section{THE MOUTH OF THE FUNNEL AND GENERAL KNOWLEDGE STATEMENTS}

The WikiRate project's starting point is its Mission Statement:

"To spur corporations to be transparent and responsive by making data about their social and environmental impacts useful and available to all".

The project's purpose has been to design and build a platform which enables its community to construct an information resource which can achieve this aim. The research component of the project has sought to answer the twinned questions of how community members can be recruited to the endeavour and how this community should work towards the goal of "better companies". Thus, the question of what type of knowledge the platform should serve could only be said to be answered once we know how a community will produce that knowledge, where that community will come from, and how that knowledge can be deployed to improve ESG performance.

The project aims to increase the transparency of companies' behaviour, and transparency is applied to data on the platform (and its provenance) and user activity (as a safeguard against manipulation). The WikiRate Mission Statement is an inscription and could be seen as the "theory-statement" driving WikiRate, where designers provide a broad definition of the user base (all), of the knowledge object (all companies) and what the project wants to achieve (make every aspect of ESG performance transparent). This is the broad mouth of the funnel of interest for WikiRate. It is the translation of this statement into the particular which funnels

\footnotetext{
${ }^{2}$ http://wikirate.org/About WikiRate
} 
knowledge creation via the design.

\subsection{Current state of ESG information}

Understanding the current state of knowledge on company ESG performance was an important first step towards designing WikiRate. The goal was to find a way for an open community-powered platform to make a positive intervention, the first question to answer was: What are the ways in which stakeholders can know about the ESG performance of companies?

Many large companies voluntarily publish annual sustainability reports: these are the source of much knowledge about their ESG performance. Companies have complete control over the content of these reports and each follows a bespoke structure, usually published as a pdf document. The Corporate Social Responsibility (CSR) teams producing these reports are often situated within the public relations part of the organisation, and improvement to the organisation's reputation is a strong motivator for reporting (Brammer \& Pavelin, 2006). Thus a sustainability report is unlikely to give a neutral and objective view on the company's sustainability.

Many large companies now report in accordance with some standard, the most used coming from the Global Reporting Initiative (GRI). In this case, one can expect to find data relating to certain indicators within the report. Proprietary services like a Bloomberg Terminal offer, to subscribers, access to data extracted from these reports and presented in a standardised machine-readable format. There is no equivalent publicly accessible resource. WikiRate aims to build an open access repository for this kind of data.

Voluntary CSR reporting is driven by stakeholder demand (Aguinas and Glavas, 2012), and WikiRate seeks to increase public engagement with these reports, demonstrating and increasing stakeholder demand. Recent years have seen the introduction of legislation which requires reporting on aspects of non-financial performance (Section 1502 of Dodd-Frank Act ${ }^{3}$, UK Modern Slavery Act 20154, European Commission non-financial reporting directive ${ }^{5}$ ). None of these examples make provision for the analysis of published reports. WikiRate aims to deploy a peerproduction approach to analyse these reports at the large scale at which they are produced.

In addition, there are questions about how available data should be used to drive improvements in performance. Proprietary ratings (e.g. KLD) have been shown to influence the performance of rated companies (Sutantoputra, 2009), and also un-

\footnotetext{
${ }^{3} \mathrm{https}: / / w w w . s e c . g o v / N e w s / A r t i c l e / D e t a i l / A r t i c l e / 1365171562058$

4 http://www.legislation.gov.uk/ukpga/2015/30/contents/enacted

5 http://ec.europa.eu/finance/company-reporting/non-financial reporting/index en.htm
} 
rated peers within a rated industry (Sharkey \& Bromley, 2015). The public at large have no insight into how these ratings are constructed or the judgments they arrive at.

Some Civil Society Organisations (CSOs) research companies' performance, offering their findings to the public as ratings and/or reports. For an individual without access to proprietary resources, these offer a way of obtaining relevant information in a form which does not require data collection or analysis. To our knowledge, there is as yet no evidence that these public ratings can influence performance in the way that proprietary ratings can. The design that WikiRate has arrived at is oriented towards such ratings, with the aim of representing existing public ratings in a common standard - but also to enable the creation of new ratings, either by borrowing aspects from existing ratings and/or by starting afresh from the raw data to produce new scoring and rating schemes.

\subsection{Stakeholders and users}

An understanding of the platform's stakeholders and potential users, and their roles in the mission to improve ESG performance, was also instrumental to the design process.

Investors and investment funds can influence companies through their investment decisions. Indications of poor ESG performance can affect companies' share prices (Amer, 2015). Offering investors more comprehensive information about ESG performance could extend the scope of this effect.

CSOs are instrumental in identifying and raising issues with ESG performance, and campaigning for change. CSOs may not have access to proprietary sources, and have limited human resources to pursue their aims. CSOs often have large numbers of members/followers who sympathise with their mission and can be mobilised. WikiRate can facilitate the work of CSOs by offering a means of engaging their followers more directly in their research effort - and by making this research accessible to a wider audience.

Academic research can yield new insights into where problems arise with ESG performance and how these can be addressed. WikiRate can facilitate novel forms of projects for students, where they study companies' reporting and other sources to identify and analyse certain pieces of information - making this data available to all.

Companies and their employees are important stakeholders as it is positive change to the practices of these companies that the WikiRate project seeks. Comparisons of the practices of competing companies can identify leaders and laggards. WikiRate aims to establish ESG performance as something that companies compete on. 
Consumers can exert influence on companies through their consumption choices. Making it easier to consider ESG performance when making these choices is a way to leverage this potential for influence. Furthermore, there is potentially great benefit in promoting wider public engagement with ESG impacts. If WikiRate can reposition these issues so they are not seen as intractable, by fostering an open community aiming to make a difference, this would be a significant development for the longerterm.

CSOs, academics and students were identified as the most important groups to serve in the early stages of the project. The role envisaged for these stakeholders is research-related, and a platform that facilitates research was deemed sufficient to recruit them, even if the platform does not yet have an established body of knowledge to offer. Other stakeholders are perceived as being more likely participants once the KA has reached a certain maturity.

\subsection{From mission-statement to hypothesis-statements and design iterations}

A set of 10 hypothesis-statements were produced, based on the broad theorymission statement inscribed on the project at its commencement, and research conducted in the early stages.

1. Statements or data about companies should be backed by reference to a publicly accessible source.

2. WikiRate is based on wiki and peer production principles, the community of users has collective responsibility for site content in general.

3. Some content types should be under the control of the user who created them or users who they authorise.

4. All user actions and user-generated content should be presented transparently with a full edit history and space for discussion.

5. It is not the role of the WikiRate team to be an arbiter of what constitutes important/irrelevant information.

6. It is not the role of the WikiRate team to be an arbiter of what constitutes $\mathrm{good} / \mathrm{bad}$ performance of companies, or the relative importance of various issues.

7. The WikiRate platform should offer a means whereby the community can assess the quality and importance of data.

8. The WikiRate platform should determine the salience of information based on community assessment of its importance, while allowing sub-communities to pursue their own approaches.

9. The WikiRate platform should be capable of both representing existing knowledge, and of facilitating the production of new knowledge.

10. The WikiRate platform should offer tools for the analysis of data, in addition to 
the collection and representation of data.

Knowledge statements 1, 2, 4, 5, and 6, have been inscribed on the project since the beginning and have remained more or less unchanged. Statements 1, 2 and 4 recognise that WikiRate's genesis is with a wiki style approach, while statements 5 and 6 re-affirm that the community of users has collective responsibility for site content. Statements 5 and 6 set the project on a path towards designing a platform for peer-production, well beyond "crowdsourcing", as all members are invited to participate as equals, self-selecting their roles.

WikiRate's approach to peer-production contains elements of lightweight (LWPP) and heavyweight peer production (HWPP) (Haythornthwaite, 2009). WikiRate embraces HWPP as the designers/owners do not take responsibility for its direction (statements 5 and 6). However, WikiRate needs to obtain one benefit of LWPP defining tasks clearly enough that users can contribute knowledge in what is a complex and contested domain. The platform has therefore been designed with features that allow some users to define tasks for others.

The first articulation of the WikiRate project was as a funding proposal, and from the beginning the platform was intended to serve both qualitative and quantitative content. In the project's first year qualitative content was prioritised, and design and development work focused on serving this. Some problems were identified with this approach. Using a wiki to construct a KA requires consensus among contributors as to what constitutes good content. The domain of company ESG performance is one where a reliance on well-established sources and 'objective facts' is limiting. Much available data comes from companies themselves, sources are not universally trusted, and the dimensions upon which ESG performance should be assessed are themselves contested. Furthermore, written accounts of corporate ESG performance are already available from a number of respected sources. At the first annual project review, a consensus emerged that WikiRate was not on track to achieve some of its aims by prioritising qualitative content. It was agreed that the project should re-orient towards quantitative data and knowledge, with a revised design. Knowledge statements 3, 7, 8, 9 and 10 were added at this point.

Statement 3 recognises that it is unlikely that a community of contributors will agree on what constitutes valid, reliable information about ESG performance. Allowing content to be "owned" allows it to exist on the platform without community consensus on its value. WikiRate's design diverges from that of a common wiki in that it focuses on quantitative data as opposed to written content, and it allows this data to be owned and managed by a specific individual or group of users. Statements 7 and 8 establish a requirement that the platform should enable the community to develop a more coherent view on companies' performance. Taken together, these three 
statements mean that WikiRate should enable independent groups/individuals with different perspectives/aims to work separately on the platform - while presenting an overview of this work to unaffiliated users which reflects the views of the community at large. The design solution for this is a voting/following system.

Statement 9 reflects an understanding that WikiRate should encapsulate and build upon existing publicly accessible knowledge about ESG performance - the more the $\mathrm{KA}$ could be pre-populated with existing knowledge, the less daunting the task of early contributors would become.

In the first year WikiRate did not offer a sufficient value proposition to attract key stakeholders as contributors. The platform has been designed to offer value to all identified stakeholders, but that value is derived from the KA which is being constructed - thus it became important to serve stakeholders who could benefit from the platform even when the KA was in its earliest stages. The platform could be of value to stakeholders like CSOs and academics if it allowed them to engage their members/followers/students in their research - offering an efficient way to produce desired data-sets and a hands-on experience of participating in research.

The validation of this change in approach stems from the fact it became much easier to engage key stakeholders as participants in the project. However, the revised design alone - moving from a qualitative to a quantitative approach - did not lead to a great change in stakeholders' response. A much warmer reception began when the project team started to populate WikiRate with data that demonstrated how this new approach was intended to function. The shift towards quantitative content also allowed for a significant volume of data to be obtained from other sources and imported to WikiRate, which served to establish the platform as a repository of information, even before a community of users was present. This echoes other notable community powered sites like Stackoverflow, where the manner in which content was produced in the early stages differs markedly from the mature stage (Mamykina et al., 2011). As with the example of Stackoverflow, WikiRate designers were among the most active contributors in the early stages, setting an example of how the platform should be used by others.

\section{FROM GENERAL STATEMENTS TO PARTICULAR DESIGN SOLUTIONS}

The desire to use quantitative data at scale puts the concept of Metrics at the centre of WikiRate's approach. Metrics are a way of asking the same question of many companies, and specifying the nature of the answers. When users add a new answer to a metric they are encouraged to make a comment which, at minimum, describes where within the cited source (e.g. a CSR report) the answer was found, along with any contextual information that supports the answer. For some metrics 
these comments are vital, potentially more useful than the answer itself. This is often the case for categorical metrics where adding an answer represents a judgment on the part of the researcher.

To take one example, the Walk Free Foundation ${ }^{6}$ have designed a set of metrics for analysis of the statements that certain companies must produce to comply with the UK's Modern Slavery Act 2015. The purpose of these metrics is to allow the Foundation to recruit volunteers for the task of analysing the thousands of statements being produced in response to this legislation. One of these metrics (see figure 3) asks whether the company identified any specific incidents related to modern slavery, with possible answers Yes and No. This metric presents an aggregate view of whether statements tend to describe the identification of incidents, while also offering a shortcut to reviewing the detail of how each statement described these (through inspection of individual answers and accompanying comments).

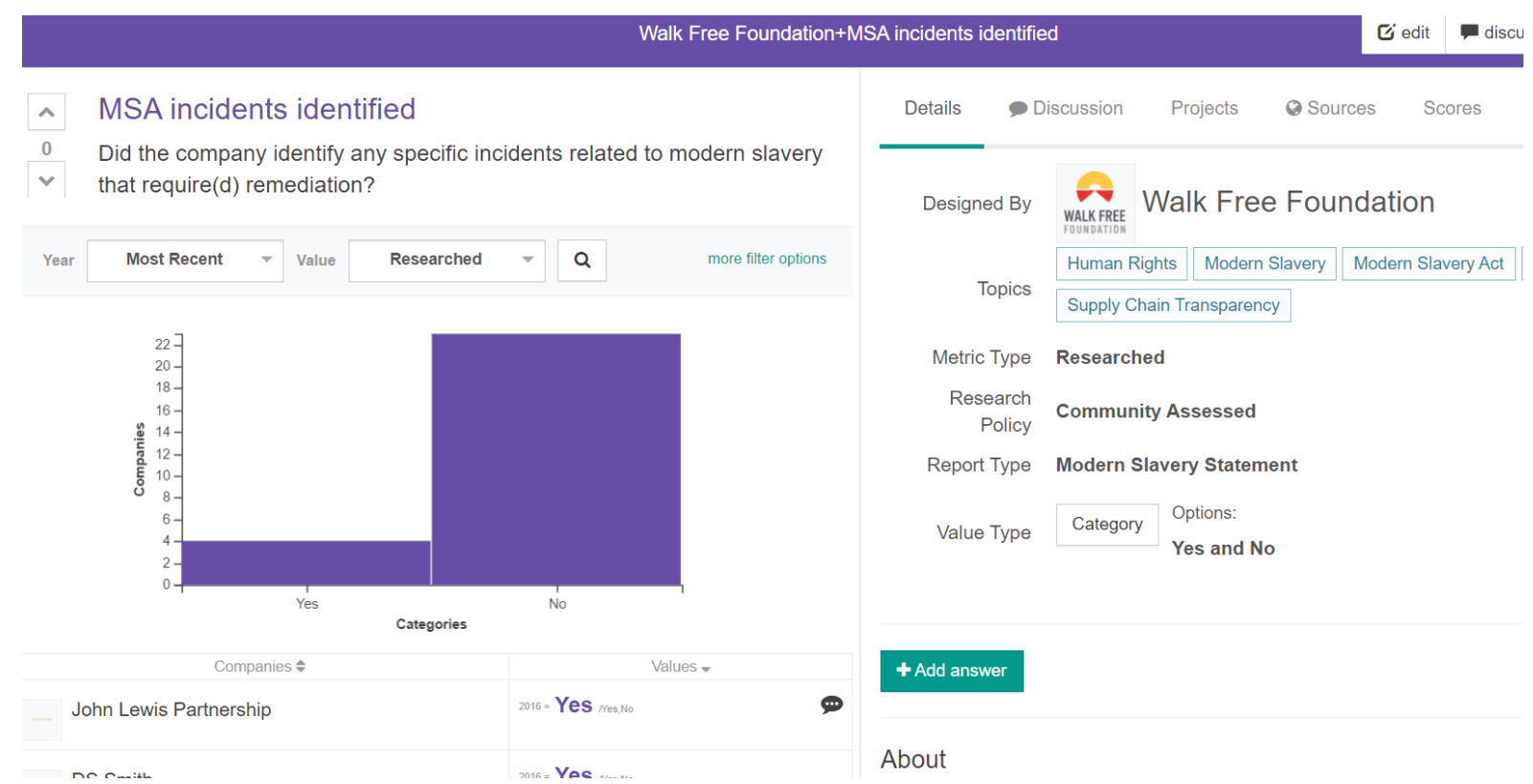

Figure 3 - Example of a researched metric page, showing on the left the question the metric asks, along with a bar chart showing aggregate answers, and the first of a list of company answers. The right side shows the metric's type, research policy, and other meta-data.

The user who creates a metric has a number of choices about how it will be used. Metrics can be used to represent data from an external source, in which case this data can be imported and the metric closed to community research. Metrics can also be used to gather new data, and the creator can decide whether to cede control of the metric and its values to the community, or to act as a moderator (by reviewing

\footnotetext{
${ }^{6}$ http://www.walkfreefoundation.org/
} 
new edits and/or data).

Metric data enters the platform either through a bulk import (when existing in machine-readable form), or added directly by users. Project and research pages have been designed to facilitate crowd research of metric data. A selection of metrics, and companies to be researched on those metrics, forms the basis of a project. From a project a "research page" for every included company is automatically generated, where metrics and their methodologies can be viewed on one side of the screen alongside relevant sources on the other (Figure 4).

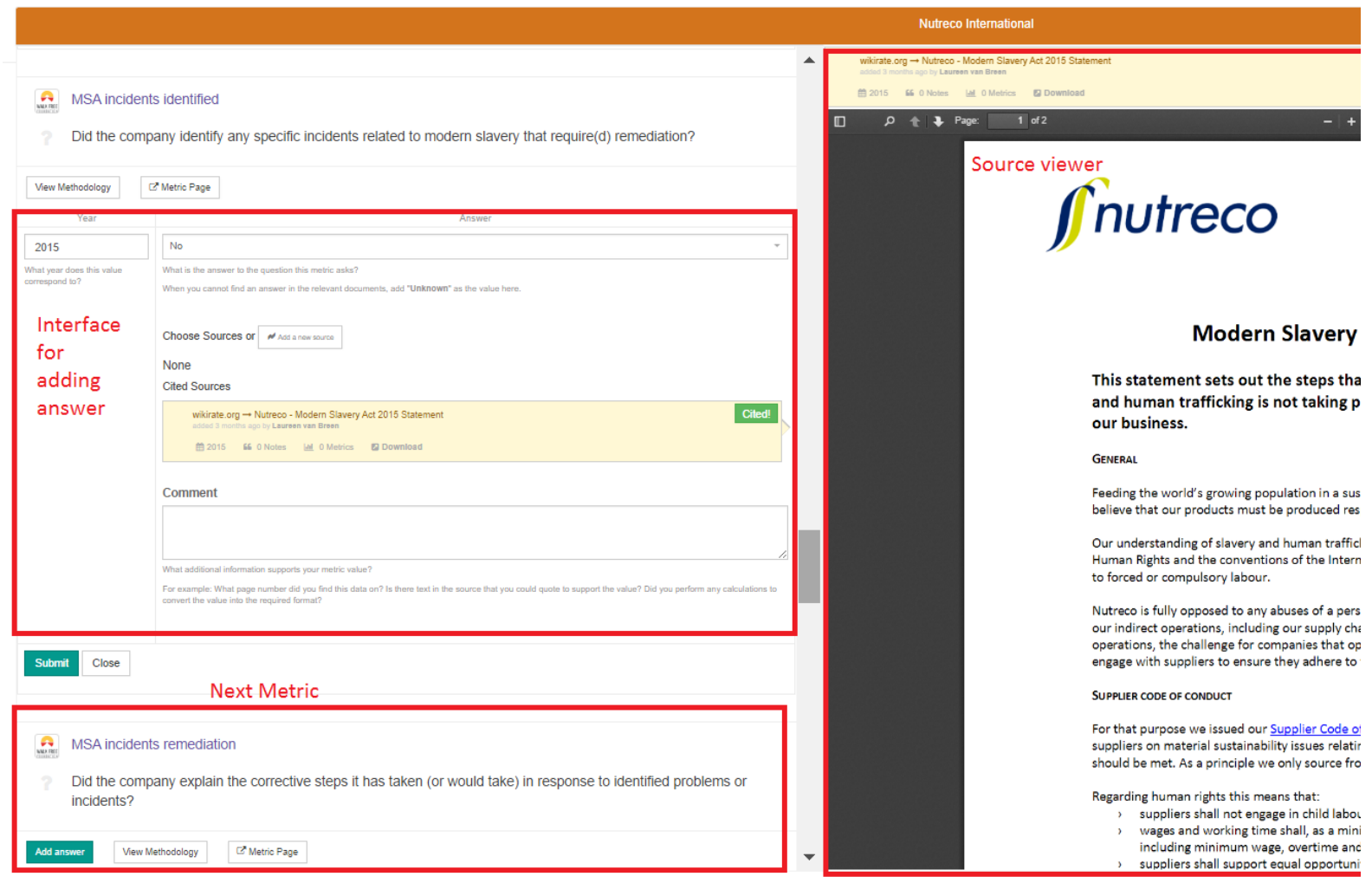

Figure 4 - The page for researching one company on a Modern Slavery Act project.

The concept of Metric as a defining feature of WikiRate seems somewhat at odds with a "Socially situated ITKA", based on a peer-production Wiki. As Metrics impose a rigid definition of knowledge and its objective representation, they are more aligned with "Representational KAs". There is a tension in WikiRate between the desire to present robust knowledge representations to some stakeholders, and the understanding that a socially situated ITKA is more appropriate as a starting point for the construction of the resource.

Although metrics are quite prescriptive and representational, the process of designing these can be a social one. A metric creator decides what its purpose will 
be. For metrics designed to facilitate peer produced data collection, the way that volunteer researchers interpret and apply these is key to assessing and improving their utility. Each metric must define the meaning of the data it holds, but also provide instructions to follow to produce reliable data. All such metrics benefit from piloting, and the review of data, comments and feedback from contributors is essential for validating the metric as a data collection instrument.

WikiRate has been designed as a peer-production platform that also encompasses 'crowdsourcing' as one way in which an individual user might contribute - if they merely complete a set data-collection task without participating in refinement of the metrics or analysis of the data, their experience will be similar to that of participating in a crowdsourcing endeavour. WikiRate goes beyond crowdsourcing however and embraces peer production, as each contributor has the potential to engage with the construction of research projects and the analysis of the data they produce.

\subsection{Governing the metric commons}

The question of what constitutes a 'good' metric is open and contested. There exists no set of metrics which are commonly accepted as reflecting all we need to know about ESG performance. Knowledge statements 5 and 6 affirm that the WikiRate project team are not to be judges of what constitutes a good or bad metric, and statements 7 and 8 call for the community to be equipped with tools for making these assessments. As metrics have become central, so have the tools that allow for their assessment and refinement.

Some of these tools stem from Wikis: these are spaces to discuss each metric and each data-point, and metric metadata and values can be collaboratively edited if allowed by their creator. Broad definitions of what constitutes a company, an ESG issue, and relevant data necessitate further means of organising metrics. To this end metrics also have topic tags, which are used to denote the issues the metric is relevant to.

It can be inferred from the statements and background that not all metrics will be "good", that there will be a differential in quality between these metrics and also a subjective element to this differential. The design solution to these issues is preference voting on metrics, whereby a user can "follow" metrics to make these part of the lens through which company performance is presented. This allows users with different interests to follow different metrics and see information that's relevant to their interests. The system for following/hiding metrics doubles as an up/down voting system - votes are aggregated to produce a score for each metric, and where a user is not signed into an account, or hasn't voted on some metrics, these scores are used to determine global metric visibility. 


\subsection{Metrics for analysis and rating}

The metrics system described thus far is central to WikiRate's approach to collecting and storing data on ESG performance. These are considered to be "researched" metrics, and each value must cite an external source as evidence (statement 1). Additionally, a class of "calculated metrics" have been designed, allowing for analyses and ratings to be constructed using data held in researched metrics. Calculated metrics can also be created by any user, the difference is that their "methodology" consists of a set of mathematical or logical operations to be performed on the data held in other metrics - they are second order metrics.

Calculated metrics serve two purposes, analysing data and building ratings. "Formula" metrics (based on Wolfram language ${ }^{7}$ ) allow for a broad set of mathematical or logical operations to be performed on data held in other metrics. For example, formula metrics can be used to reproduce existing analyses like the Center for Sustainable Organizations' context-based carbon metric ${ }^{8}$ which contextualises a company's carbon emissions with their economic contributions.

Scores and WikiRatings offer a standardised way of constructing ratings, they are designed to be easily understood and interacted with, while capable of reproducing the kind of ratings/benchmarks that some NGOs produce. A Score metric maps the values held in any other metric onto a $0-10$ value scale ( 0 is bad, 10 is good). A WikiRating takes a set of Scored metrics and produces a weighted average, the "methodology" for a WikiRating is simply the list of input scores and their weightings.

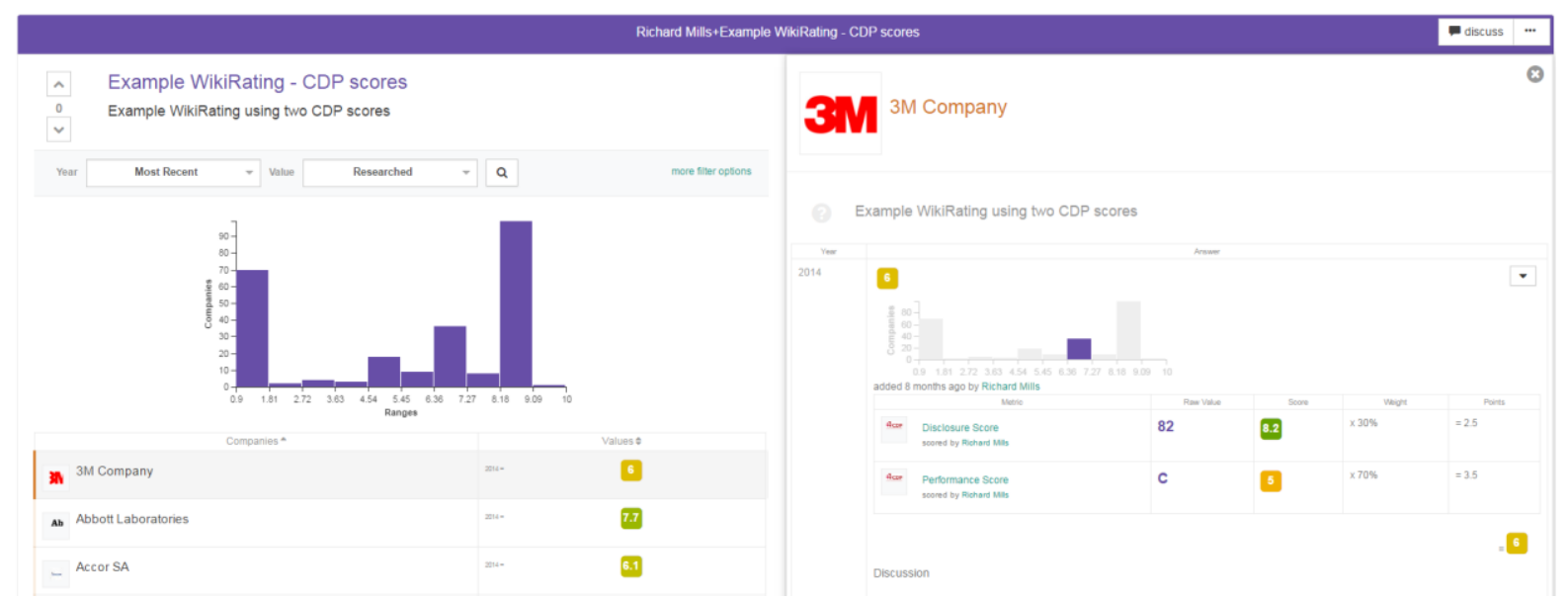

Figure 5 - Example of a WikiRating with two input metrics

Each Score metric is independent of the metric it is scoring - multiple users can create their own Scores that interpret data in different ways. Scores can also be

\footnotetext{
${ }^{7}$ http://reference.wolfram.com/language/

$8 \underline{\text { http://www.sustainableorganizations.org/context-based-metrics-in-public-domain.html }}$
} 
applied to the outputs of Formula metrics. WikiRatings can incorporate other WikiRatings, as well as Scores. The intention with this system is to make analyses and ratings as modular as possible, allowing them and their constituent parts to be "re-mixed" with other ratings, and to encourage users to "play" with the data and produce personalised ratings of company performance.

Ratings are seen as a key method of influencing company performance and thus they feed back to the general mission statement of WikiRate. Presenting ratings from established credible sources on the same platform in the same format is seen as a way to increase their power. Allowing any user to interact with these ratings, pick them apart and create their own from the components, is a way to increase the reusability of the data and to engender deeper public engagement. WikiRate aims to empower users (individual and institutional) to get involved at this high level of rating companies on their behaviour - and to deepen their knowledge and engagement with the issues through doing so. The preference/voting system for metrics is intended to enhance these dynamics, offering some competition around producing the "best" new ratings or metrics.

\subsection{Some Partnership Examples and Knowledge Generation with the KA}

Knowledge generation via WikiRate currently happens mainly through partnerships with key stakeholders. These partnerships have been fostered by the design team and remain vital to the viability of WikiRate. Here we briefly mention some relevant examples. WikiRate's collaborators include organisations like Amnesty International (crowdsourcing analysis of conflict minerals reports), the Global Reporting Initiative (to represent their standard accurately as metrics), the United Nations Global Compact and Principles of Responsible Management Education (PRME). The partnership with PRME is nearing the end of a pilot round in which 9 PRME signatory Universities have used WikiRate to set up and run research projects investigating companies' performance in relation to the UN Sustainable Development Goals. This pilot is ongoing at time of writing, over 1,000 participating students have so far collected 22,400 data-points from the reports of 539 different companies - with some engaging in the analysis of this data and some going further to contact companies directly about what they did or did not disclose in their reports.

This kind of data-oriented dialogue between companies and their stakeholders is vital for WikiRate to achieve its ultimate objective of "better companies". Research to understand what individual companies are disclosing can be thought of as the foundation of the Knowledge Artefact, a foundation upon which the community can begin to construct analyses and comparisons, and to reason about how companies' performance should be measured, what could or should be disclosed.

With WikiRate's foundational tools and structures in place, and data-sets that are 
poised to scale, the project's attention is now turning to engaging and enrolling additional stakeholders that will be important in achieving the end goal - investors, journalists and consumers. If WikiRate becomes a platform through which these stakeholders find out about a company's ESG performance, companies are more likely to pay attention and become active participants themselves. The plan to engage these stakeholders is to have data that they cannot obtain easily elsewhere and to present this in forms which make it accessible and attractive. The story of WikiRate's development thus far has been largely about developing the platform to facilitate the collection of this data. With this data resource now growing steadily, attention is turning to the development of tools for analysis of this data (through calculated metrics and visualisations) and for embedding it in other sites (through API access but also analyses that can be embedded elsewhere) to extend its reach.

\section{Discussion and Conclusion}

In this paper we presented our direct experience in the design of the WikiRate platform, a KA whose goal is to improve the ESG performance of companies. We have offered an interpretative framework for understanding how ESG knowledge creation and the design of WikiRate are connected via a funnel of interest (Law, 1986). In this process a general mission statement and a set of detailed knowledge statements have been translated into particular actionable design solutions by our team. We have also shown how the concept of the funnel of interest allows one to see that in WikiRate there is an interplay between situativity in the community building and objectivity of the knowledge being represented. Thus if we were to locate WikiRate in Cabitza and Locoro's (2014) map (Figure 1), this could be seen as a CSCW situated project which projects itself toward the objectivity axis. WikiRate's funnelling approach seeks to create interest and enable communities of contributors to engage in the social construction of knowledge about ESG performance, and for those same communities to then produce an objective representation of that knowledge which can influence the behaviour of other stakeholders. To serve the aims of the project, this knowledge does not have to be a perfect representation of the facts, it merely has to rival or complement alternative (proprietary) sources of such knowledge in quality or scope. In this scenario, the WikiRate community could exert some influence over the behaviour of companies, by establishing a prioritised set of questions about ESG performance and making the answers available to investors and other stakeholders in accessible forms.

In the process of designing WikiRate we learned a number of lessons which may benefit the wider community of KA designers and practitioners. In this discussion we offer a set of design and community building recommendations for practitioners who may be working on projects of similar scope and ambition to WikiRate. 
The first observation we can make is that the process of building WikiRate did not take place in a linear way, there has been considerable back and forth both in the design and in the relations with a variety of stakeholders. Therefore, a recommendation for design practitioners is that even if the overarching process of building a KA may be seen as the translation and funnelling of general mission statements into particular design solutions, the plan that designers adopt for enacting this translation has to be flexible and open to adjustments and negotiations as they move forward. Traditionally the design methodologies of ITs assume a linear form, divided into sequential steps (Avison and Fitzgerald, 1995). It is known that linear methodologies do not allow much flexibility as they need to fulfil requirements defined at the very beginning of a project. In our experience, general statements for a project may be defined at the beginning, but there may be multiple emerging directions and design solutions for achieving the goals set in mission statements. Flexibility has been fundamental in our experience, not only in terms of designing specific features but also in terms of defining and achieving the immediate aims of the project. Among other things, the project made a major pivot away from a focus on qualitative content and towards the quantitative after the first year. This pivot involved designing tools for the collection (researched metrics) and analysis (calculated metrics) of quantitative data, and although both sets of tools were designed at the time, development efforts have been directed to refining the data collection tools in response to observations of their use. A choice was made in favour of continuing to work on the foundational data collection aspects, at the expense of delaying development of the analysis tools that would allow the data to be leveraged.

The ambition of the project has had a significant impact on WikiRate's design, and it was the project's ambition which lead to a peculiar interplay of situativity and objectivity. Thus a second recommendation for designers coming from the WikiRate experience is that the degrees of situativity and objectivity of a KA - as enacted through a design - should be seen as instrumental for achieving the mission of a KA. Designers thus may need to take strategic decisions as to what the roles of objective and situated knowledge are, and how the interplay between these can be harnessed. In WikiRate, the fact that the project aims to assess all companies on any aspect of ESG performance is important, as is the ultimate goal of not just understanding but improving behaviour. Assessing all companies on any aspects is the broad mouth of the funnel of interest in WikiRate. The WikiRate platform as KA is itself instrumental for achieving the mission statement and the scale of the project thus has called for certain design and community building decisions. The nature of the subject matter and background of contributors means that much of the knowledge generated is socially situated, while the need to offer reliable and structured information to key stakeholders calls for a representational approach to design.

WikiRate cannot yet be considered a success when considered in relation to the 
project's ambitious aim of improving corporate ESG performance globally. Indeed, there is only anecdotal evidence that some companies are aware of WikiRate. However, WikiRate is a long-term endeavour which still necessitates attention and effort for pursuing its aim, even if the design of platform's data collection aspect is now reasonably mature. Thus, a third recommendation is that the process of community building and translation into practice of the mission statement is a continuous one - in which the KA's design might take a detour to interest certain stakeholders, whose presence and contributions may subsequently make it possible to interest a further set of stakeholders. The design of the KA may therefore not immediately translate into the expected knowledge generation at the end of a funnel of interest. Looking back at Figure 2 (the funnel of interest), the project has achieved the first three steps (crystallising a general mission statement and core design statements, as well as creating particular reliable and usable design solutions), but it still is in the process of building the exit of the funnel and generating a substantial body of knowledge. The design team is aware that achieving the global impact that WikiRate aims for is likely to take some years. To achieve the project's aim, companies must not only be aware of WikiRate but also perceive the account the platform gives of their performance to be important enough to warrant their direct engagement - disclosing additional answers to metric questions and responding to existing data-points or the discussion around them.

A fourth recommendation for community building is to see the value of members of the project team engaging directly on the platform as community members themselves. When the first iteration of the data collection tools were deployed the active use of these tools by members of the project team served both to identify improvements and to seed the platform with examples of their use. Once live examples could be demonstrated, this greatly improved the success of outreach efforts to potential institutional users. The degree of collaboration between the design team and these institutional users has also been greater than expected. The concept of metrics and projects that could be defined by an organisation and opened up to community research was appealing, but execution was not intuitive. Through these collaborations we as a team have also learned how to better structure and support research projects on the platform for other actors, and discovered how the platform can be improved to better meet the needs of each partner organisation.

To conclude, during the design and community building processes, the biggest lesson of all has been a shift in our understanding of WikiRate's purpose for achieving its mission statement. This was conceived initially as a platform that could grow and support a community to understand and improve corporate ESG performance. We think of it now as a platform to bring together the efforts of existing groups working towards this end, to amplify the impact of these groups and engage a greater number and diversity of participants in this effort. 


\section{REFERENCES}

Aguinis, H. and Glavas, A., (2012), What we know and don't know about corporate social responsibility a review and research agenda. Journal of management, Vol. 38 No. 4, pp. 932-968.

Akrich, M., (1992), The de-scription of technical objects. In: Bijker W. and Law J. eds. Shaping technology building society, (pp. 205-224). Cambridge, MA: MIT Press.

Amer, E., (2015), The penalization of non-communicating UN Global Compact's companies by investors and its implications for this initiative's effectiveness.

Business \& Society. http://dx.doi.org/10.1177\%2F0007650315609303

Avison, D.E. and Fitzgerald, G., (1995), Information Systems Development: Methodologies, Techniques and Tools, $2 d$ ed. New York: McGraw-Hill.

Brammer, S. J. and Pavelin, S., (2006), Corporate reputation and social performance: The importance of fit. Journal of Management Studies, Vol. 43 No. 3 , pp. $435-455$.

Bruns, A., (2008), Blogs, Wikipedia, Second Life, and beyond: From production to produsage (Vol. 45). New York: Peter Lang.

Cabitza, F. and Locoro, A. (2014), "Made with Knowledge" - Disentangling the IT Knowledge Artifact by a Qualitative Literature Review. In KMIS 2014, pp. 64-75.

Callon, M., (1984), Some elements of a sociology of translation: domestication of the scallops and the fishermen of St Brieuc Bay. The Sociological Review, Vol. 32 No. 1, pp. 196-233.

Callon, M., (1991), Techno-economic networks and irreversibility. In Law, J., editor, A sociology of monsters. Essays on power, technology and domination, (pp. 132161). London: Routledge.

Ciborra, C. and associates, (2000), From Control to Drift: The Dynamics of Corporate Information Infrastructure. Oxford: Oxford University Press.

Ciborra, C., (2002), The labyrinths of information: Challenging the wisdom of systems: Challenging the wisdom of systems. Oxford: Oxford University Press.

Cockburn, A., and Highsmith, J., (2001), Agile software development, the people 
factor. Computer, Vol. 34 No. 11, pp. 131-133.

Davenport, T. H., De Long, D. W., and Beers, M. C., (1998), Successful knowledge management projects. Sloan management review, Vol. 39 No. 2, pp. 43-57.

De Paoli, S., and D'Andrea, V., (2008), How artefacts rule web-based communities: practices of free software development. International Journal of Web Based Communities, Vol. 4 No. 2, pp. 199-219.

Gherardi, S., (2000), Practice -based Theorizing on Learning and Knowing in Organizations. Organization, Vol. 7 No. 2, p. 211-223.

Haythornthwaite, C. (2009), Crowds and communities: Light and heavyweight models of peer production. In System Sciences, 2009. HICSS'09. 42nd Hawaii International Conference on (pp. 1-10). IEEE.

Hevner, A. R., March, S. T., Park, J., \& Ram, S., (2004), Design science in information systems research. MIS quarterly, Vol. 28 No. 1, pp. 75-105.

Howe, J., (2006), “The Rise of Crowdsourcing,” Wired, June 2006.

Junior, R. M., Best, P. J., and Cotter, J., (2014), Sustainability reporting and assurance: a historical analysis on a world-wide phenomenon. Journal of Business Ethics, Vol. 120 No. 1, pp. 1-11.

Kogut, B. M. and Metiu, A., (2001), Open source software development and distributed innovation. Oxford Review of Economic Policy, Vol. 17 No. 2, pp. 248 264.

Lanzara, G. F., and Morner, M. (2005), Artifacts rule! How organizing happens in open source software projects. In Czarniawska B. and Hernes T. (Eds.). ActorNetwork Theory and Organising, (pp. 67-90). Copenaghen: Copenhagen Business School Press.

Latour, B., (1987), Science in action: How to follow scientists and engineers through society. Cambridge, MA: Harvard university press.

Latour, B., (1991), Technology is Society made durable. In Law, J. (Ed.) A Sociology of Monsters, (pp. 103-131). London: Routledge \& Kegan Paul.

Laufer, W. S., (2003), Social accountability and corporate greenwashing. Journal of Business Ethics, Vol. 43 No. 3, pp. 253-261. 
Law, J., (1986), The Heterogeneity of Texts. In Callon M., Law J. and Rip A. (Eds.) Mapping the dynamics of Science and Technology, (pp. 67-83). London: The Macmillan press Itd.

Levitt, B., and March, J.G. (1988), Organizational learning. Annual review of sociology, Vol. 14 No. 3, pp. 319-338.

Lin, Y., (2005),The Future of Sociology of Floss. First Monday, Special Issue \#2: Open Source, October 2005, http://www.firstmonday.org/issues/special10 10/lin/index.html

Mamykina, L., Manoim, B., Mittal, M., Hripcsak, G., and Hartmann, B., (2011), Design lessons from the fastest q\&a site in the west. In Proceedings of the SIGCHI conference on Human factors in computing systems (pp. 2857-2866). ACM.

Mills, R. and Fish, A., (2015), A computational study of how and why reddit. com was an effective platform in the campaign against sopa. In International Conference on Social Computing and Social Media (pp. 229-241). Springer International Publishing.

Mills, R., De Paoli, S., Diplaris, S., Gkatziaki, V., Papadopoulos, S., Prasad, S.R., McCutchen, E., Kapadia, V. and Hirche, P., (2016), WikiRate.org - Leveraging collective awareness to understand companies' environmental, social and governance performance. In International Conference on Internet Science (pp. 7488). Springer International Publishing.

Nonaka, I. and Takeuchi, H. (1995), The knowledge-creating company. New York: Oxford University Press

Norman, D. A., (1993), Cognition in the head and in the world: An introduction to the special issue on situated action. Cognitive science, Vol. 17 No. 1, pp. 1-6.

Raymond, E.S., (1998), The Cathedral and the Bazaar, http://www.openresources.com/documents/cathedral- bazaar/

Sharples M, Hogg D., Hutchinson C., Torrance S. and Young, D., (1989), Computers and Thought. A practical Introduction to Artificial Intelligence. Cambridge,MA: MIT Press.

Sharkey, A. J., and Bromley, P., (2014), Can ratings have indirect effects? Evidence from the organizational response to peers environmental ratings. American Sociological Review, Vol. 80 No. 1, 63-91.

Simon, H. A., (1996), The sciences of the artificial. Cambridge MA: MIT press. 
Suchman, L., (1987), Plans and Situated Action. New York: Cambridge University Press.

Sutantoputra, W. A., (2009), Social disclosure rating system for assessing firms' csr reports. Corporate Communications: An International Journal, Vol. 14 No. 1, pp. 3448.

Wenger, E., (2000), Communities of practice and social learning systems.

Organization, Vol. 7 No. 2, pp. 225-246.

Winograd, T. and Flores, F., (1986), Understanding Computer and Cognition. A New Foundation for Design. Norwood,NJ: Ablex Publishing. 